\title{
AUV Online Mission Replanning for Gap Filling and Target Inspection
}

\author{
Juan David Hernández, Eduard Vidal, Jennifer Greer, Romain Piasco, \\ Patrick Jaussaud, Marc Carreras, and Rafael García
}

\begin{abstract}
In most of the current operational autonomous underwater vehicles (AUVs), a survey mission is generally composed of two main stages. The first one conducts an exhaustive coverage over an area of interest, while gathering data of the sea bottom. Then, and after processing the collected data, a second mission is programmed to obtain more detailed information of potential targets, and to cover the gaps that resulted from the first exploration. However, this two-survey strategy can be inefficient, since it requires establishing a communication link between the AUV and its operator for retrieving the data and reprogramming the second mission. To cope with this situation, we present a mission planner that endows an AUV with the capability of extending its missions online. With our approach, the vehicle is also required to conduct an initial and predefined survey of an area of interest, but it processes the gathered data onboard to plan 3D feasible paths to complement the initial exploration. To validate our approach, we present real-world results with the AsterX AUV.
\end{abstract}

\section{INTRODUCTION}

Nowadays, autonomous underwater vehicles (AUVs) have become a standard tool for seabed mapping. In most of the current operational AUVs, the mission plan is programmed in advance, so that the vehicle can afterwards dive and autonomously cover the predefined area from a constant and safe altitude. Such mission plans are specified by an operator, and commonly consist of the path to be followed and a series of payload commands, which establish when and where sensors must acquire different data along the pre-planned route. The nature of the data mainly depends on the mission objectives, as well as the sensors installed in the vehicle. The most commonly used sensors include multibeam echo sounders (MBES), side scan sonars (SSS), sub-bottom profilers (SBP), optical cameras, and physicochemical sensors.

In this approach of covering a predefined area, the preplanned route is generally not modified during the survey. Instead, the objective is to sweep the area in order to detect and localize potential targets, which can include geological formations [1], shipwrecks [2], and other artificial or natural underwater structures [3]. A common approach in this kind of missions is to conduct a first exhaustive survey, and then

Work on this paper has been supported by the EUROFLEETS FP7 Project under the Grant agreement ID: 312762. J.D. Hernández, E. Vidal, M. Carreras, and R. García are with the Underwater Vision and Robotics Research Center (CIRS), University of Girona, Spain. juandhv@eia.udg.edu, rc4559@gmail.com, marc.carreras@udg.edu, rafael.garcia@udg.edu. J. Greer, R. Piasco, and P. Jaussaud are with the L'Institut Franais de Recherche pour l'Exploitation de la Mer (Ifremer), Toulon, France. \{jennifer.greer, romain.piasco, patrick.jaussaud\}eifremer.fr extract and analyse the gathered data to program a second and more specific survey. This latter one attempts to obtain more details about the potential targets, as well as to cover possible gaps resulted during the first exploration. However, this two-survey strategy can be inefficient, since it requires establishing a communication link between the AUV and its operator (located in the mother ship) for retrieving the data and reprogramming a new mission. This is particularly unnecessary in modern AUVs with long-term autonomy.

Aiming to overcome some of the aforementioned limitations, we propose to endow an AUV with a mission planner, or high-level controller, that extends its decisionmaking capabilities. This additional control layer must allow the vehicle not only to conduct predefined surveys, but also to autonomously detect and inspect potential subareas of interest without the need of resurfacing. To do so, the AUV is assumed to be equipped with a looking-downward multibeam sonar, which gathers data from the sea bottom along the initial mission. This data can be then automatically processed onboard the AUV in order to detect anomalies and gaps, which are marked as regions that require further inspection, thus enlarging the original survey. In the case of anomalies (i.e., objects that protrude above the surrounding seafloor), the vehicle must conduct closer explorations; while in the case of gaps, it must complete covering the area that was initially defined.

To validate our approach, we used the proposed online mission planner within the AsterX AUV (see Fig. 1) to conduct survey missions over areas of interest. Furthermore, some of the test surveys were intentionally planned to leave gaps, thus fully demonstrating the new AUV capabilities. Results include in-water tests conducted with the AsterX AUV with real targets during a campaign in the bay of $\mathrm{La}$ Ciotat, France, in October 2016.

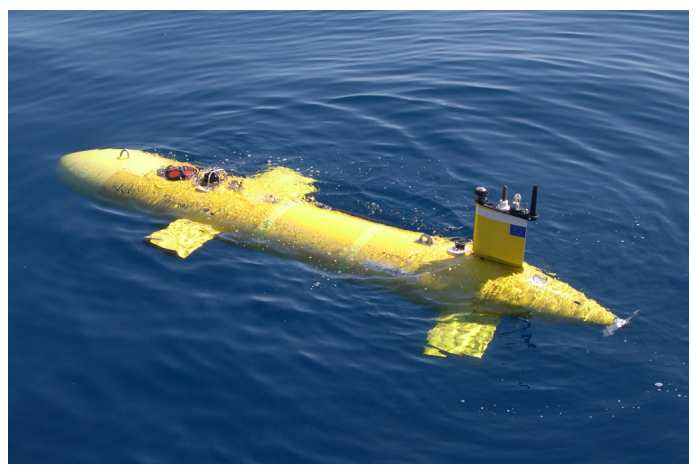

Fig. 1: AsterX AUV 


\section{ONLINE MISSION REPLANNING WITH THE ASTERX AUV}

The AsterX AUV software architecture is composed of three main functional blocks. 1) The AUV low-level controller, also referred to as frontseat, that guides the vehicle using the automated control engine (ACE) middleware. This controller is executed over QNX operating system, thus guaranteeing real-time computation constraints. Furthermore, this functional block also handles the vehicle's navigation and safety routines. 2) The backseat controller that extends the vehicle's capabilities and applications by easing the implementation of high-level routines, such as algorithms for path/motion planning, path-following, and docking. The backseat controller has its own dedicated computer that works under the robot operating system (ROS) (over Linux). 3) The payload controller that acts as a bidirectional interface between the interoceptive and exteroceptive sensors, and both the frontseat and backseat controllers. Figure 2 depicts the AsterX's software architecture, including its three functional modules, and how they communicate to each other.

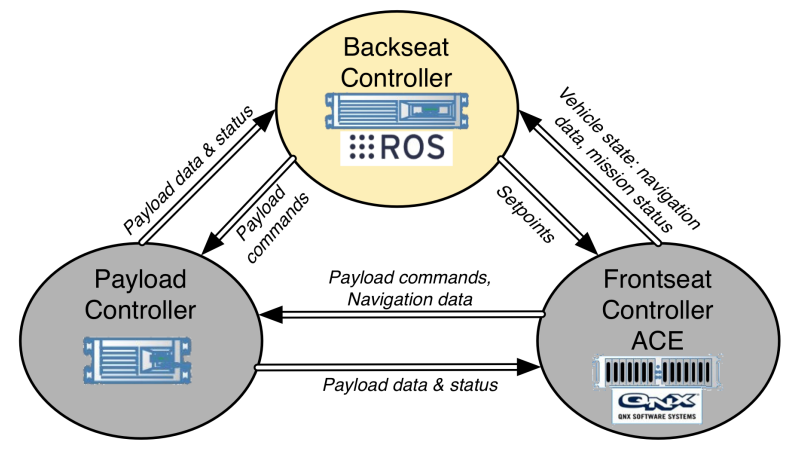

Fig. 2: AsterX AUV software architecture

Having this software architecture in mind, the online mission planner can be developed within the backseat. This allows including different specialized processes, or nodes as they are referred to in ROS. The proposed approach includes four nodes: a) the target and gap detector, b) the mission handler, c) the path planner, d) and the path-following controller (see Fig. 3). It is also important to notice that these nodes not only are capable of exchanging information between each other, but also can externally communicate with the frontseat and payload controllers.

In the AsterX AUV, the initial and pre-planned part of the survey is directly programmed in the frontseat. This can be done by using a set of primitives that allow defining missions that are composed of a series of waypoints and payload commands. Once the vehicle has completed this initial survey, the frontseat informs the backseat to take over control of the vehicle. To do so, this latter one has a limited time to start sending setpoints to the vehicle's lowlevel controller. Otherwise, the frontseat will start conducting an idle manuever, which consists of describing a circular trajectory until the mission timer indicates that the vehicle must go back to the surface.

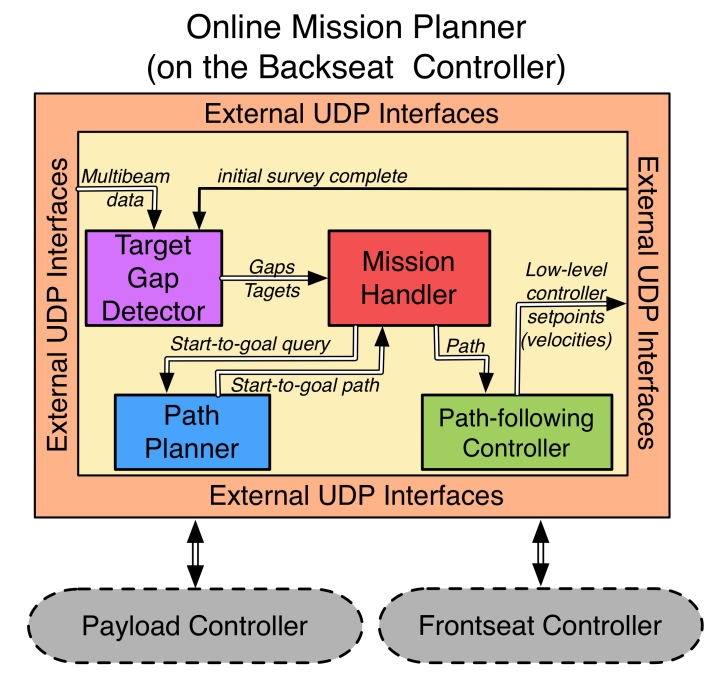

Fig. 3: Nodes, or computational processes, of the online mission planner that is implemented on the backseat controller.

\section{A. Gap and Target Detector}

While the AsterX AUV is conducting the initial survey defined within the frontseat, the backseat, and especially the target/gap detector, receives the multibeam data when it is indicated in the mission plan. Once the backseat is informed of the initial survey's end, the target/gap detector proceeds to analyse the gathered multibeam data. This process seeks to identify potential targets (anomalies) and gaps, including their positions and approximate dimensions.

This node uses a geometric approach to identify the potential targets. To do so, it first approximates the seabed shape by using a grid of planes. These planes are extracted from the received point cloud by executing random sample consensus (RANSAC) [4]. Then, all detections that are far enough (i.e., over a pre-defined threshold) from the estimated seabed are clustered, thus defining the potential targets' regions. Finally, a bounding box is fitted to each cluster that contains a minimum amount of detections.

On the other hand, the whole explored area is discretized into a grid that is used to identify the gaps. Then, the amount of multibeam data detections is stored for each cell in the grid. Finally, those cells with a density of detections lower than a pre-established threshold are reported for further inspection. Once both the potential targets and gaps have been identified, their position and bounding boxes are sent to the mission handler.

\section{B. Mission Handler}

This node works as a mission coordinator. It waits until receiving a list of the targets and gaps' positions. Around such positions, this node establishes subareas of interest that require to be further covered. To do so, the mission handler defines a series of consecutive start-to-goal queries to first explore the potential targets in close proximity, and then to cover the gaps at the same altitude of the initial survey. Such queries are sent to the path planner, which computes the 3- 
dimensional (3D) and feasible AUV paths that extend and complement the mission.

While such paths are being calculated, the mission handler sends a straight-line path to the path-following controller. This permits to start sending setpoints to the low-level controllers, thus maintaining control over the vehicle. The length of this initial path is equivalent to the time required to obtain the first query solution, which must guide the vehicle to approach the first target or gap. Once the planner starts providing the solution paths, they are sent to the pathfollowing controller.

\section{Path Planner}

This node calculates the 3D solution paths for the startto-goal queries that are provided by the mission handler. An important aspect of this path planner is the necessity of taking into account the vehicle motion constraints. The AsterX, for instance, is a torpedo-shaped AUV subject to horizontal and vertical motion constraints. Since its propulsion force is located in the backside, the vehicle cannot conduct purely lateral motion. Instead, it can only change its direction of motion by moving forward or backward. Furthermore, its vertical motion is also limited to a maximum descending/ascending speed. In order to deal with these constraints, this node uses the Optimal RRT (RRT*) [5], which is a sampling-based path-planning algorithm.

\section{Path-following Controller}

Once the planner has provided the 3D paths, the mission handler sends them to the path-following controller. This node implements an extended version of the line-of-sight (LOS) path-following controller as described in [6]. Essentially, the following operations are performed iteratively:

- The position of the robot is projected into the path.

- An intermediate goal is generated some meters ahead of the projected point.

- The orientation controller turns the vehicle to point towards this intermediate goal, and the surge speed is set to the constant value used in the path planner.

Finally, the vehicle stops when it reaches a pre-defined region of acceptance around the end of the path.

\section{RESULTS}

In order to validate the proposed mission planner, the AsterX AUV conducted a real-world in-water trial using the backseat controller with the aforementioned nodes (see Fig. 4). The mission consisted in detecting and navigating in close proximity to a plane wreck that is located in the bay of La Ciotat, France. Before starting the mission, the AsterX was programmed to follow a predefined coverage survey of the area of interest. This initial survey was defined within the frontseat controller to navigate at $10 \mathrm{~m}$ deep and with a constant surge speed of $1.5 \mathrm{~m} / \mathrm{s}$. Once completed this part, the frontseat handed over the control to the backseat.

Figure 5 depicts the area of interest, the initial survey, and the potential target and gaps detected from the multibeam data. It can also be observed that after completing the initial

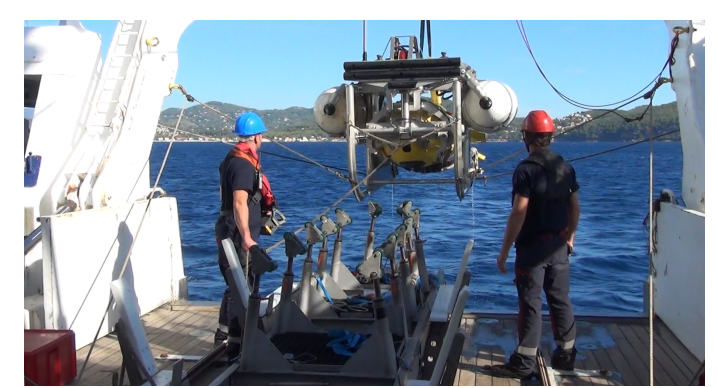

Fig. 4: AsterX AUV deployment from the ship l'Europe

survey, the backseat guided the vehicle over a straight-line trajectory. This maneuver was conducted at the same speed and safe depth, while the target/gap detector processed the multibeam data to detect the potential targets and gaps, if any of them existed.

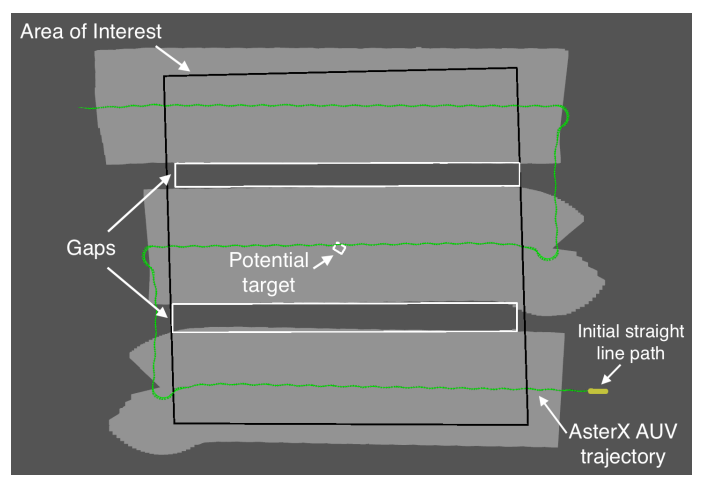

Fig. 5: Potential target and gaps detected after completing the initial survey with the AsterX AUV. It can be observed the area of interest in black, the AUV trajectory in green, the area covered with multibeam sonar with light grey, and two gaps and one potential target in white.

After the detector provided the locations of the potential targets and gaps, the path planner calculated a complementary 3D trajectory to both explore the potential targets and cover the gaps. For the former case, the planner calculated a path $15 \mathrm{~m}$ over than highest point detected in the target. For the latter case, the planner defined paths to cover the gaps at the depth of the initial survey, i.e., $10 \mathrm{~m}$ for this particular mission. As the extended mission required navigating at different altitudes, the planner calculated 3D paths that met the ascending and descending motion constraints. Once this calculation was completed, the whole path was sent to pathfollowing controller. Figure 6 depicts the extended mission to navigate over the potential target and to cover the gaps.

Since the depth for further exploring the targets was different than the one used to cover the gaps, this kind of missions requires planning 3D AUV paths. Figure 7 depicts how the AsterX AUV further the target by following a calculated path. To do so, the vehicle descended to a specified depth with respect to the detected anomaly (target), while conducting turning maneuvers. Both horizontal and vertical motions were calculated to meet the vehicle capabilities. 


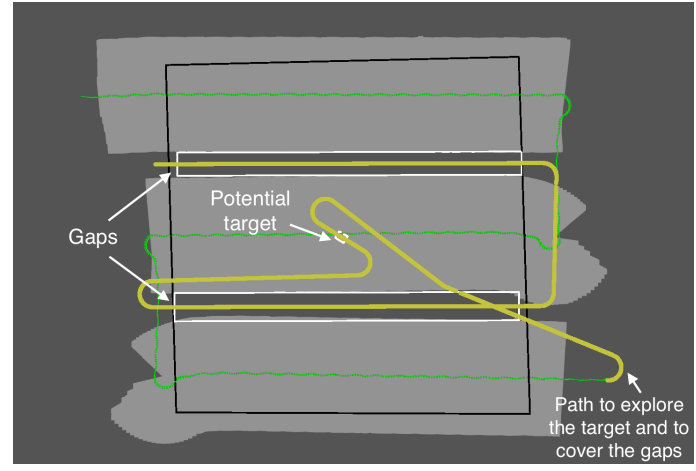

Fig. 6: Path planned to further cover the potential target and gaps with the AsterX AUV. It can be observed the new extended mission in yellow. Although the view is from the top, the path is $3 \mathrm{D}$ since it requires different altitudes to inspect targets and to cover gaps.

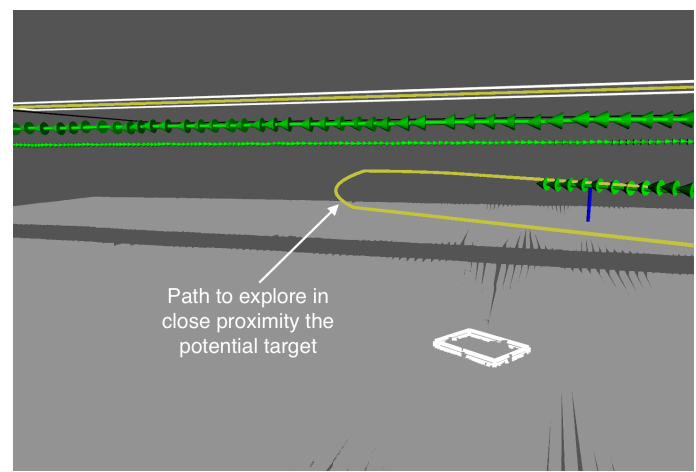

Fig. 7: The AsterX AUV approaches to further inspect a potential target. The vehicle trajectory is presented in green, while the calculated path appears in yellow.

Finally, Figure 8 shows the final AUV trajectory after completing the whole mission. It can be observed how the vehicle not only travelled over the target, but also covered the gaps.

\section{CONCLUSIONS}

We presented a mission planner that endows an AUV with the capability of extending online a survey path. To do so, the AUV first conducts a pre-defined coverage of an area of interest, while it gathers information of the sea bottom. Then, the vehicle automatically processes onboard the collected data to identify potential targets and gaps. If they exist, the proposed planner extends the mission to complement the exploration. The whole process is done without surfacing, thus avoiding establishing a communication link between the AUV and its operator. This approach was successfully validated with the AsterX AUV in a real-world survey mission during a campaign in the bay of La Ciotat, France.

\section{REFERENCES}

[1] E. Galceran, V. Djapic, M. Carreras, D. P. Williams, and U. Robotics, "A Real-time Underwater Object Detection Algorithm for Multi-beam Forward Looking Sonar," 2012.

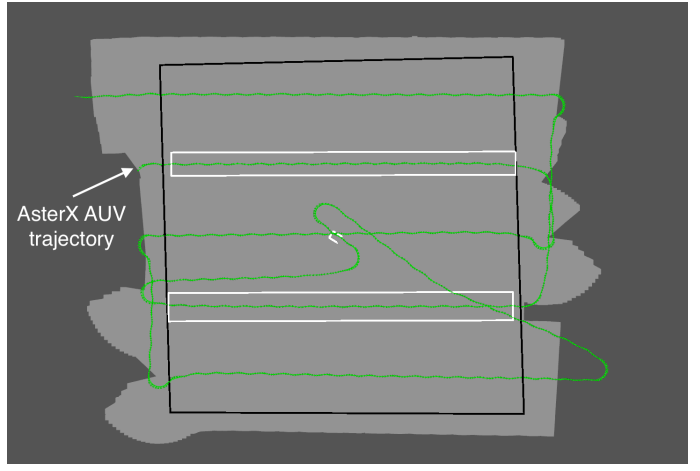

Fig. 8: The AsterX AUV completed the mission by inspecting the potential target and covering the gaps from the initial survey. It can be observed the area of interest in black, the AUV trajectory in green, the area covered with multibeam sonar in light grey.

[2] N. Gracias, P. Ridao, R. Garcia, J. Escartn, M. L'Hour, F. Cibecchini, R. Campos, M. Carreras, D. Ribas, N. Palomeras, L. Magi, A. Palomer, T. Nicosevici, R. Prados, R. Hegeds, L. Neumann, F. de Filippo, and A. Mallios, "Mapping the moon: Using a lightweight auv to survey the site of the 17th century ship 'la lune';", in MTS/IEEE OCEANS Bergen, pp. 1-8, June 2013.

[3] J. Escartin, R. Garcia, T. Barreyre, M. Cannat, N. Gracias, and A. Shihavuddin, "Optical methods to monitor temporal changes at the seafloor: The Lucky Strike deep-sea hydrothermal vent field (MidAtlantic Ridge)," 2013.

[4] M. a. Fischler and R. C. Bolles, "Random Sample Consensus: A Paradigm for Model Fitting with Applicatlons to Image Analysis and Automated Cartography," Communications of the ACM, vol. 24, no. 6, pp. $381-395,1981$.

[5] S. Karaman and E. Frazzoli, "Sampling-based Algorithms for Optimal Motion Planning," The International Journal of Robotics Research, vol. 30, pp. 846-894, jun 2011.

[6] T. I. Fossen, Handbook of Marine Craft Hydrodynamics and Motion Control. Chichester, UK: John Wiley \& Sons, Ltd, apr 2011. 